progressed (toppling to the right or left would be random due to the external bilateral body symmetry).

The results of Babcock and Robison, however, suggest that curling was not bilaterally symmetrical but skewed to favour retraction on the animal's left, thus raising and exposing to attack the righthand margin of the trilobite's body.

If curling did indeed commence at the tail-end and was skewed to favour retraction on the animal's left, then the righthand margin of the pygidium and posterior thoracic segments would have been exposed first and for the longest period. One might therefore expect to see a higher frequency of sublethal predation scars in these areas. Interestingly, the three examples shown by Babcock and Robison are all of injuries to the right-hand region of the pygidium and/or posterior thorax.

\section{Department of Physiology,}

DAVID P. MAITLAND

Medical School,

The University of the Witwatersrand, Johannesburg, South Africa 2193.

SIR-Babcock and Robison show that for their sample of Palaeozoic trilobite fossils, there is a marked tendency for healed injuries to occur on the right side rather than the left, and they interpret this as evidence of behavioural asymmetry in the fossil record, support for an early evolutionary history for brain laterality ${ }^{1}$. I would suggest an alternative, simpler explanation. Trilobites may have possessed a biological asymmetry that put a soft vital organ on their left side, as in the case of the human heart, making attacks to that side more likely to be fatal. Simply put, it may be that trilobites were attacked equally often on both sides, but more of those attacked on the right side survived to join Babcock and Robison's sample.

An example of such a phenomenon occurred during the Second World War. The US military studied fighter planes returning from missions to try to improve their survival rate and were considering adding heavy armour to those parts of the plane that tended to show the greatest concentration of hits from enemy fire, until statisticians pointed out the fallacy of that argument ${ }^{3}$. The more vulnerable parts of the plane were those with the fewest hits; planes hit there tended not to return at all. The single most vulnerable part, the pilot's head, was without serious scar in the sample of planes that returned. STEPHEN STIGLER

Department of Statistics,

University of Chicago,

5734 University Avenue,

Chicago, Illinois 60637, USA

1. Babcock, L.E. \& Robison, R.A. Nature 337, 695-696 (1989).

2. Meglitsch, P.A. Invertebrate Zoology (Oxford University Press, London, 1967).

3. Wallis, W.A. J. Am. Statist. Ass. 75, 334-335 (1980)

\section{Quasar lensing by galaxies?}

SIR-We wish to point out that the extraordinary statistical alignments reported by Webster et al. ${ }^{1}$ between low-redshift galaxies and high-redshift quasars, if explained as a gravitational lensing effect, can be used to place a robust lower limit on the total cosmological density. We also amplify a point raised by those authors, namely that, although the effect is expected to occur, the quantitative assumptions that one has to make to explain such a strong effect are inconsistent with our present understanding of galaxies. We thus disagree with Canizares' assessment ${ }^{2}$; in our opinion these alignments may indeed be "too close for comfort".

According to the lensing hypothesis, the images of high-redshift quasars experience an amplification when they are seen through foreground galaxies; this amplification allows one to see intrinsically fainter quasars, which are more numerous. Taking Webster et al.'s model at face value, suppose that there is an amplification of about a factor of two for the fraction $f \approx 0.01$ of the sky that lies within 6 arcsec of galaxies above their magnitude limit. The distance of galaxies at a redshift $z$ is about $r=z c / H$, where $H$ is Hubble's constant, and for their sample Webster et al. quote $z \approx 0.3$. Gravitational amplification by a factor of two with this geometry requires a mean surface density of lensing material of $\Sigma \approx 0.3 \mathrm{~g} \mathrm{~cm}^{-2}$, whether the lensing amplification is due to 'microlensing' by individual stars or to the galactic potential as a whole ${ }^{3}$. From this information alone we can estimate the mean density contributed by this lensing population; in terms of the cosmic density parameter, $\Omega$,

$$
\Omega_{1} \approx \frac{8 \pi G f \Sigma \theta^{2}}{H z c} \approx 0.15 \theta^{2} h^{-1}
$$

where $\theta^{2}$ is the ratio of the actual lens area to the 6-arcsec circle, and $h=H / 100$ $\mathrm{km} \mathrm{s}^{-1} \mathrm{Mpc}^{-1}$; according to current estimates, $h$ should lie between 0.5 and $1 . \Omega_{\text {, }}$ is a lower limit on the mean density contributed by material projected within about $16.5 \theta h^{-1} \mathrm{kpc}$ of a galaxy, for $z=0.3$.

This density estimate is consistent with some other measurements of cosmic darkmatter density, but the dark matter here is much too close to the luminous parts of the galaxies to be consistent with other dynamical mass measurements. Based on estimates of the luminosity density of all galaxies, the mass-to-light ratio required to achieve the above mean density estimate is about $M / L \approx 240 \theta^{\circ}$ in solar units (independent of $h$ ). On no scale are dynamical estimates of mass ${ }^{+}$consistent with such a large $M / L$. The range of $M / L$ found for the inner parts of ordinary galaxies $(\theta \approx 1)$ is between 5 and 20 , even allowing for the presence of a massive halo; on the scale of groups and clusters $(\theta \approx 20)$ dynamical measurements range up to $M / L \approx 400 h$, far short of what is required on this scale. On this and still larger scales, the density of the lenses is not consistent with limits on the overall deceleration of the Universe.

Unusual alignments or structures do not resolve this dilemma; if only a small fraction of galaxies contributes to the effect, then the quasar surface density enhancement required by the observations is correspondingly higher, and the problems get worse. Therefore, within a conventional understanding of galactic systems we can find no model to explain the large enhancement seen by Webster et al.

\section{Craig J. HOGAN RAMESH NARAYAN SIMON D.M. WHITE}

Steward Observatory,

University of Arizona,

Tuscon, Arizona 85721, USA

WEBSTER ET AL. REPLY-We agree with the conclusions of Hogan, Narayan and White, that the alignments we reported could suggest that the mass associated with galaxies is greater than has been previously estimated. However, the data in ref. 1 demonstrate a highly significant detection of an overdensity with a 20 lower bound of 1.7. Estimating $\Omega_{1}$ using this lower bound significantly lowers the value calculated.

The calculated overdensity will vary with $\theta$, and thus so too will estimates of the mass. Our data relate to the average overdensity for radii of 4-6 arcsec. We note that the surface mass density implied for the galaxies includes a contribution from any 'halo' component which may extend to many tens of kiloparsecs.

We are in the process of substantially increasing the sample size, obtaining galaxy redshifts, and obtaining CCD images of all quasar-galaxy associations. These data should greatly improve our estimates of the size of the effect and hence of the mass required for lensing.

RACHEL L. WeBster

Astronomy Department,

University of Toronto,

Toronto M5S 1A1, Canada

Paul C. HewetT MARgARET E. HARDING

Institute of Astronomy,

Madingley Road,

Cambridge CB3 OHA, UK

Dartmouth College,

GARY A. WEGNER

New Hampshire 03755, USA

\footnotetext{
Webster, R.L., Hewett, P.C., Harding, M.E. \& Wegner, G.A. Nature 336, 358-359 (1988)

Canizares, C.R. Nature 336, 309-310 (1988)

2. Canizares, C. R. Nature 336, 309-310 (1988).

4. Faber, S.M. \& Gallagher, J.S. A. Rev. Astr. Astrophys. 17 135-188 (1979).
}

NATURE · VOL $339 \cdot 11$ MAY 1989 